\title{
Epidemiological trends
} and sociodemographic factors associated with acute hemorrhagic conjunctivitis in mainland China from 2004 to 2018

\author{
Rong Liu' ${ }^{1 \dagger}$, Yuxing Chen ${ }^{2 \dagger}$, Hao Liư ${ }^{2}$, Xihui Huang ${ }^{3}$ and Fang Zhou ${ }^{2 *}$
}

\begin{abstract}
Background: Acute hemorrhagic conjunctivitis (AHC) is classified as a class $C$ notifiable infectious disease in China and poses a great threat to public health. This study aimed to investigate the epidemiological trends and hotspots of AHC in mainland China. Sociodemographic factors that could contribute to early warning of AHC were further explored.
\end{abstract}

Methods: Yearly and monthly incidences of acute hemorrhagic conjunctivitis by date and region from 2004 to 2018 were extracted from the Data Center of China Public Health Science. Joinpoint regression and spatial autocorrelation analysis were performed to explore the epidemiological trends and hotspots of AHC. A generalized linear model was then applied to explore the relationship between sociodemographic factors and AHC incidence.

Results: The average annual AHC incidence was 3.58/100,000 in mainland China. The first-level spatial and temporal aggregation areas were distributed in Guangxi, Hainan, Guangdong, Guizhou, Hunan, Jiangxi, Fujian, Chongqing, Hubei, Anhui, and Zhejiang, with gathering times from 2010/1/1 to 2010/12/31 (RR =20.13, LLR=474,522.89, $\mathrm{P}<0.01)$. After 2010, the $A H C$ incidence was stable (APC $=-8.37,95 \% \mathrm{Cl}:-23.02-9.06)$. However, it was significantly increased in low- and middle-income provinces (AAPC $=10.65,95 \%$ Cl: $0.62-21.68$, AAPC $=11.94,95 \%$ Cl: 0.62-24.53). The peak of AHC occurred during the August to October period. Children who age $0-3$ years are identified as highrisk group with $\mathrm{AHC}$ incidence significantly increased (APC $=31.54,95 \% \mathrm{Cl}: 0.27-72.56)$. Birth rate, population ages 0-14 (\% of total population), passenger traffic, and urban population (\% of total population) were positively associated with the AHC incidence, while per capita gross domestic product was negatively associated with the AHC incidence.

Conclusion: Overall, the AHC incidence was stable after 2010 in China, but it was significantly increased in low- and middle-income provinces. Regions with a high birth rate, population ages 0-14 (\% of the total population), passenger traffic, urban population (\% of the total population) and low per capita gross domestic product are at high risk of

\footnotetext{
*Correspondence: zfant8385@163.com

${ }^{\dagger}$ Rong Liu and Yuxing Chen have contributed equally to this work

${ }^{2}$ Institute of Chronic and Non-Communicable Disease Control and Prevention, Hubei Provincial Center for Disease Control

and Prevention, No. 35 Zhuodaoquan North Road, Hongshan District, Wuhan 430079, China

Full list of author information is available at the end of the article
} permits use, sharing, adaptation, distribution and reproduction in any medium or format, as long as you give appropriate credit to the original author(s) and the source, provide a link to the Creative Commons licence, and indicate if changes were made. The images or other third party material in this article are included in the article's Creative Commons licence, unless indicated otherwise in a credit line to the material. If material is not included in the article's Creative Commons licence and your intended use is not permitted by statutory regulation or exceeds the permitted use, you will need to obtain permission directly from the copyright holder. To view a copy of this licence, visit http://creativecommons.org/licenses/by/4.0/. The Creative Commons Public Domain Dedication waiver (http://creativeco mmons.org/publicdomain/zero/1.0/) applies to the data made available in this article, unless otherwise stated in a credit line to the data. 
incidences of AHC. In the future, public health policy and resource priority for AHC in regions with these characteristics are necessary.

Keywords: Acute hemorrhagic conjunctivitis, Trends, Disease hotspots, Sociodemographic factors

\section{Introduction}

Acute hemorrhagic conjunctivitis (AHC) is an acute viral eye disease that was first reported in Ghana in 1969 [1]. It is mainly caused by coxsackievirus A24 variant (CA24v) and human enterovirus type 70 (EV70), and it is characterized by bilateral eye pain, increased secretion, keratitis, and conjunctival congestion, commonly known as "pinkeye" [2]. Generally, the majority of cases present obvious symptoms but result in a favorable prognosis and rarely with severe complications or fatal infections [3-5]. Due to the rapid onset of symptoms, short incubation period and high infectivity, it usually leads to an outbreak or epidemic in the short term [6].

Since the first occurrence of AHC (caused by CA24v) in Singapore in 1970 [7], it has spread rapidly around the world. In recent decades, more than 10 million AHC cases have been reported worldwide [8], including several large outbreaks in Asia and South America. In 2002, the largest outbreak of AHC was reported in South Korea, with over 1,000,000 cases [9]. In 2014, an unprecedented nationwide outbreak of infectious conjunctivitis occurred in Thailand, which affected more than 300,000 individuals over 3 months [10]. In 2017, it was first reported in Mexico with 10,000 cases and then spread to other neighboring countries in the Caribbean and in South America, resulting in large outbreaks [2]. For instance, over 200,000 cases of AHC occurred in Brazil during the summer of 2017/2018 [13].

The economic and medical burden of conjunctivitis is also noteworthy. In France, during the 13-week epidemic, the estimated cost of the outbreak was $€ 3,341,191$ [14]. In the United States, 28\% of annual emergency department visits are associated with conjunctivitis, amounting to approximately 560 million dollars in costs [15].

Although there has been no specific estimation of AHC costs in China to date, it still poses a great threat to public health. China has been classified as the most AHC-affected country worldwide [16]. Since the first cases were confirmed in 1971, many AHC cases have regularly appeared [17]. There have been 2 large outbreaks in different provinces. In 2007, the largest AHC epidemic in China was reported in Yunnan, which caused 74,263 cases [5]. Three years later, 69,635 cases caused by CA24v were reported in Guangdong [18]. To date, the AHC incidence ranked fifth among Class C notifiable diseases in 2020 [19].
Several studies have been performed to illustrate the epidemiological characteristics of AHC in China. However, most of the studies performed space-time analysis of AHC in a region-specific manner [20,21]. Whether epidemiological trends of AHC change during long-term epidemics and what sociodemographic factors contribute to AHC has rarely been studied. Determining these questions is important because it helps to understand the dynamics of AHC and to determine where AHC is severe. Due to the lack of vaccines, identifying the sociodemographic factors associated with AHC could be integrated into the current surveillance system for early warning and risk prevention of AHC. To address these data gaps, we analyzed the long-term trends across time and space for $\mathrm{AHC}$ in mainland China using national notifiable infectious surveillance data from 2004 to 2018 . We subsequently developed models to estimate the sociodemographic factors associated with AHC incidence.

\section{Materials and methods Data sources Acute hemorrhagic conjunctivitis surveillance data}

In this study, yearly and monthly AHC incidences (per 100,000) were applied for analysis, defined as the number of annual (monthly) incident cases divided by the population size [22]. The data were obtained by region from 2004 to 2018 from the Data Center of China Public Health Science, a publicly available webbased database established by the Chinese Center for Disease Control and Prevention (CDC) [23], which summarizes and sorts 31 provinces/municipalities/ regions and national notifiable infectious surveillance data from the National Disease Supervision Information Management System (NDSIMS). This notifiable infectious disease routine reporting system was first established by the Chinese government in the 1950s and has been web-based since 2003, covering all populations in mainland China [22]. Physicians and local health authorities are required to register in this system within $24 \mathrm{~h}$ of diagnosis [24]. AHC incidence data retrieved from this system were also collected from all reported cases, and the definition of AHC was in accordance with the national diagnostic criteria based on the epidemiological history, clinical symptoms, and laboratory conjunctival cytology examination. (1) Epidemiological history: Most patients had a clear history of contact infection through eye-hand, object, 
and water-eye contact. Epidemiological history easily causes epidemics or outbreaks, commonly in the summer and autumn, and epidemic periods without seasonality. (2) Clinical symptoms, such as foreign body sensation in the eye, ocular redness, ocular stinging, photophobia, lacrimation and frequent discharge. (3) Laboratory examination: EV70 or CA24v viruses were isolated from conjunctival swabs or conjunctival scraping cultures and identified as EV70 or CA24v viruses by microneutralization tests. Indirect immunofluorescence techniques were used to detect EV70 or CA24v antigen in conjunctival smears or cell culture smears. Serum anti-ev70 or anti-ca24v antibody titers in recovered patients were more than 4 times higher than those in acute patients $[2,25]$.

\section{The sociodemographic factors associated with acute hemorrhagic conjunctivitis}

We aggregated the following list of sociodemographic factors from 31 provinces from the China National and Provincial Statistics Yearbook that were hypothesized to be associated with incidences of AHC, which has been demonstrated in previous studies [26-28]: birth rate, urban population (\% of total population), population density, population ages $0-14$ (\% of total population); passenger traffic; per capita gross domestic product; and health workers (per 1000 people). These factors were chosen after a literature review of AHC and other infectious disease-related studies, also considering the data availability. Specifically, we hypothesized that population characteristics are an important factor affecting the spread of infectious disease; thus, we included birth rate, urban population (\% of the total population) and population density in the model, which has been documented to be related to various infectious disease outbreaks [29-31]. In addition, based on previous AHC studies [18, 32, 33] and from the results of our joinpoint regression showing that young children are high-risk age groups, variable population ages $0-14$ (\% of the total population) was also included. Passenger traffic variable was included to represent transportation factors since modern transportation systems has been improving, promoting regional exchanges and mobility, which also promoting the spread of disease [34]. Per capita gross domestic product is one of the most common economic research factors that has been well documented in infectious or noninfectious diseases [31, 35], while AHC diagnosis, treatment, prevention, control and reporting are all directly based on the capacity of the health system [29, 34]. In this study, we used health workers (per 1000 people) to represent health system-related factors.

\section{Statistical analysis}

\section{Spatial autocorrelation analysis}

Retrospective spatiotemporal scan statistic

The retrospective spatiotemporal scan statistic [37] was applied to detect the space-time cluster of AHC based on the discrete Poisson model. Dynamic and two-dimensional spatiotemporal was established to scan the incidence of disease in 31 provinces/municipalities/regions. A movable cylindrical window was created in the map to scan cases, with the size and position of the window in dynamic change, and to calculate the difference in incidence between the area inside and outside the window. The HO hypothesis was that disease is randomly distributed in space and time. The alternative hypothesis (H1 hypothesis) assumed that, compared to outside, the incidence of disease increased inside the scanning window. The differences in incidence between regions inside and outside the scanning window were calculated and tested by the log likelihood ratio (LLR) [38]. The scanning window with the largest LLR was identified as a high incidence cluster window. Study areas contained in the high incidence cluster window were found, and the relative risk (RR) was calculated. Monte Carlo simulation was used to assess whether LLR was significantly different from zero.

\section{Spatial autocorrelation analysis}

Global and local spatial autocorrelation analyses were used to measure spatial autocorrelation [39]. This paper used global Moran's I to detect the degree of spatial autocorrelation of AHC incidence and bivariate Moran's I to test the spatial autocorrelation between sociodemographic factors and AHC incidence from the whole region. In addition to above global Moran's I measurements, the Anselin local Moran's I was also used to reflect the local spatial correlations within geographic units to consider the existence of spatial heterogeneity and thus to identify the hot/cold spots and outliers. Based on the fact that the spatial correlation may differ locally within a large study area [40].

For the results of local spatial autocorrelation analysis, Anselin local Moran's I presented four categories of results: high-high cluster (high AHC incidence area surrounded by high AHC incidence area), low-low cluster (low AHC incidence area surrounded by low AHC incidence area), high-low cluster (high AHC incidence area surrounded by low AHC incidence area), and low-high cluster (low AHC incidence area surrounded by high AHC incidence area) [41]. The value of Moran's I ranges from -1 to 1 ; specifically, if Moran's $I>0$, there exists a positive spatial correlation; the larger this value, the stronger the correlation will be. Conversely, if Moran's 
$\mathrm{I}<0$, negative spatial correlation exists. The $\mathrm{Z}$ test and $\mathrm{P}$ values were used to assess significant differences.

\section{Joinpoint regression analysis}

The joinpoint regression model [42] was used to examine the age distribution of $\mathrm{AHC}$ and its trends from 2004 to 2018 in mainland China. We further divided 31 provinces/municipalities/regions into 3 income groups (high-, middle-, low-income) by its ranks of per capita gross domestic product in 2018 [43]. Using the commonly used division method tertile to divide the ranks into three equal sections, the ranges $0-33.3 \%$, 33.4$66.7 \%, 66.8-100 \%$ were defined as high-, middle- and low-income groups, respectively. The annual percentage change (APC) with its $95 \%$ confidence interval (CI) was calculated for each identified trend segment. The $\mathrm{Z}$ test was used to assess whether the APC was significantly different from zero. Significant APC used the terms "increase/decrease" to describe trends. Meanwhile, for nonsignificant APC, the term "stable" was used. We also estimated the average annual percentage change (AAPC) to describe long-term trends [29], assuming there was only 1 segment for the full range of our study period (2004-2018).

\section{Generalized linear model}

The log-linear generalized linear model was used to fit the AHC incidence and 7 sociodemographic factor covariates during 2004-2018. The details are as follows:

Log - linear : $\lg ($ AHC incidence $) \sim \operatorname{norm}\left(\lg (\mathrm{u}), \sigma^{2}\right)$, where $\lg (\mathrm{u})=\beta_{0}+\beta_{1^{*}}$ Year $+\beta_{2^{*}}$ Birth rate $+\beta_{3^{*}}$ (Population ages $0-14$ (\% of total population $))+\beta_{4^{*}}$ Urban population (\% of total population) $+\beta_{5^{*}}$ Population density $+\beta_{6^{*}}$ lg Passenger traffic $+\beta_{7^{*}}$ Per capita Gross domestic product $+\beta_{8^{*}}$ (Health workers (per 1000 people)), $\sigma^{2}=$ variance of $\lg (\mathrm{AHC}$ incidence).

The best-fitting model was determined according to the Akaike information criterion [44].

\section{Software}

Microsoft Excel 2016 was used for data extraction, sorting, and cleaning, and SaTScan (version 9.5), Joinpoint (version 4.8.0.1), and R (version 3.6.1) were used for further data analysis. A brief outline of the study is shown in Additional file 1: Table S1.

\section{Results}

The incidence of acute hemorrhagic conjunctivitis in mainland China from 2004 to 2018

In total, 720,640 cases were reported in mainland China from Jan 1, 2004 to Dec 31, 2018. The average annual incidence was $3.58 / 100,000$. The top five incidence regions of AHC were Guangxi (20.91/100,000), Hainan $(17.68 / 100,000)$, Guangdong $(9.84 / 100,000)$, Chongqing $(7.04 / 100,000)$, and Hubei $(6.34 / 100,000)$ provinces (Fig. 1, Table 1).

The space-time cluster of acute hemorrhagic conjunctivitis in mainland China from 2004 to 2018

The global and local spatial autocorrelation analysis results demonstrated a positive correlation of AHC incidence in mainland China. High-high aggregation areas were often found in South China (Additional file 1: Table S2 and Additional file 1: Fig. S1). Two spatial and temporal aggregation areas were revealed. The first-level spatial and temporal aggregation areas were distributed in Guangxi, Hainan, Guangdong, Chongqing, Hubei, Zhejiang, Guizhou, Hunan, Jiangxi, Fujian, and Anhui, with gathering times from $2010 / 1 / 1$ to $2010 / 12 / 31$ $(\mathrm{RR}=20.13$, $\mathrm{LLR}=474,552.89, \quad P<0.01)$. The secondary spatial and temporal aggregation areas were Beijing and Tianjin from $2004 / 1 / 1$ to $2004 / 12 / 31 \quad(R R=1.23$, LLR $=22.52, P<0.01)($ Fig. 1$)$.

\section{The incidence trends of acute hemorrhagic conjunctivitis} in mainland China from 2004 to 2018

The joinpoint regression model revealed that the AHC incidence remained stable after $2010(\mathrm{APC}=-8.37$, 95\% CI: -23.02 to $9.06, P<0.05$ ). From 2004 to 2010 , the incidence of AHC presented a significant increasing tendency, with an APC of 34.57 (95\% CI: 2.75-76.24, $P<0.05$ ) (Fig. 2a). From 2004 to 2018, 13 provinces showed significant increasing trends, including Hainan, Hubei, Yunnan, Anhui, Gansu, Hubei, Henan, Shandong, Jiangxi, Hebei, Inner Mongolia, Shaanxi, and Qinghai Guizhou, while 4 provinces showed significant decreasing trends, including Beijing, Tianjin, Shanghai and Xinjiang (Table 1, Fig. 2c).

When sub grouped by income level, the incidences of AHC in low and middle-income provinces were significantly increased (AAPC $=10.65,95 \%$ CI $0.62-21.68$; $\mathrm{AAPC}=11.94,95 \%$ CI $0.62-24.53, P<0.05)$ (Fig. 2b).

\section{The seasonal and age group distribution of acute} hemorrhagic conjunctivitis in mainland China from 2004 to 2018

We found a clear seasonality of AHC incidence, with one peak of AHC appearing from August to October (Fig. 3a). When examining age groups, children had a higher average annual incidence, especially in the age group of 3 years $(9.40 / 100,000)$. AHC incidence was increased in the age of 0 to 3 (APC $=31.54,95 \%$ CI: $0.27-$ 72.56, $P<0.05)$, while after the age of 3 , it was decreased $(\mathrm{APC}=-7.58,95 \% \mathrm{CI}:-8.77-6.38, P<0.05)$ (Fig. 3b). 


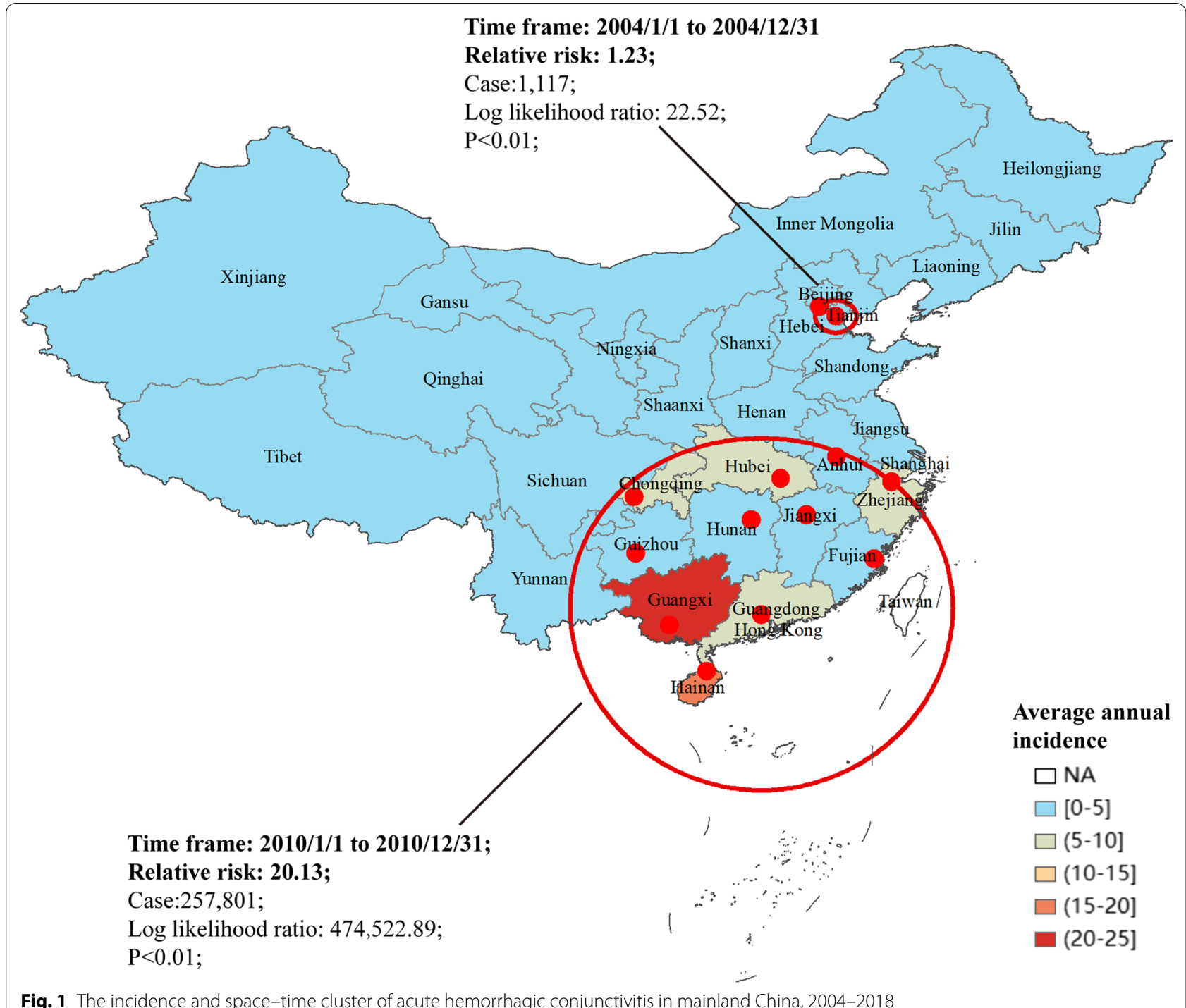

Sociodemographic factors associated with the incidence of acute hemorrhagic conjunctivitis

Bivariate global spatial autocorrelation analysis and GLM both explored the association between sociodemographic factors and AHC incidence from different perspectives. The GLM results revealed that a high birth rate $(\beta=18.51, P<0.001)$, population ages $0-14$ (\% of the total population) $(\beta=0.08, P<0.05)$, urban population (\% of the total population) $(\beta=0.08$, $P<0.001)$, and passenger traffic $(\beta=0.12, P<0.001)$ were positively associated with AHC incidence, while a low per capita gross domestic product $(\beta=-0.0004$, $P<0.001)$ was negatively associated with AHC incidence (Table 2). The bivariate global spatial analysis results were mainly consistent with GLM results (Additional file 1: Table S3).

\section{Discussion}

Understanding the epidemiological distribution and specific spatiotemporal patterns of infectious diseases, also identifying its associated risk factors are the most important task for preventing and controlling infectious diseases [22]. In this study, we illustrate epidemiological trends, hotspots and sociodemographic factors associated with AHC in mainland China from an epidemiological and public health standpoint by using longterm population-based surveillance data. We reported that the epidemiological trends of AHC incidence significantly increased from 2004-2010 but stabilized after 2010. The incidence of AHC has its own characteristics. We found that the burden of AHC was relatively high in South China, particularly in Guangxi, Guangdong and Hainan provinces, and it was significantly increased in 
Table 1 Incidence and trends of acute hemorrhagic conjunctivitis by 31 surveillance provinces/municipalities/regions in mainland China, 2004-2018

\begin{tabular}{|c|c|c|c|c|c|c|}
\hline \multirow[t]{2}{*}{ Region } & \multirow[t]{2}{*}{ Number of cases } & \multicolumn{3}{|c|}{ Average annual incidence $(/ 100,000)$} & \multirow[t]{2}{*}{ AAPC } & \multirow[t]{2}{*}{ Trends } \\
\hline & & 2004-2018 & 2004-2009 & 2010-2018 & & \\
\hline Guangxi & 150,716 & 20.91 & 6.45 & 30.55 & $8.49(-3.45$ to 21.91$)$ & Stable \\
\hline Hainan & 23,477 & 17.68 & 4.00 & 26.8 & $31.80(9.54-58.58)^{*}$ & Increased \\
\hline Guangdong & 143,833 & 9.84 & 7.21 & 11.6 & $1.67(-12.17$ to 17.69$)$ & Stable \\
\hline Chongqing & 30,686 & 7.04 & 4.06 & 9.03 & $3.32(-6.58$ to 14.27$)$ & Stable \\
\hline Hubei & 54,810 & 6.34 & 2.03 & 9.22 & $19.95(4.06 \text { to } 38.28)^{*}$ & Increased \\
\hline Zhejiang & 49,228 & 6.32 & 4.55 & 7.50 & $-4.47(-16.76$ to 9.65$)$ & Stable \\
\hline Anhui & 31,888 & 3.48 & 0.54 & 5.45 & $24.58(12.48 \text { to } 37.98)^{*}$ & Increased \\
\hline Yunnan & 23,438 & 3.36 & 1.85 & 4.37 & $26.12(7.77 \text { to } 47.60)^{*}$ & Increased \\
\hline Sichuan & 40,429 & 3.29 & 2.77 & 3.64 & -7.13 ( -17.59 to 4.67$)$ & Stable \\
\hline Hunan & 29,099 & 2.97 & 1.26 & 4.11 & $10.69(-2.02$ to 25.06$)$ & Stable \\
\hline Shaanxi & 15,943 & 2.82 & 0.64 & 4.28 & $11.77(-8.72$ to 36.87$)$ & Stable \\
\hline Guizhou & 13,599 & 2.5 & 2.3 & 2.63 & $8.46(0.56 \text { to } 16.99)^{*}$ & Increased \\
\hline Jiangxi & 16,009 & 2.39 & 0.62 & 3.56 & $15.29(2.78 \text { to } 29.32)^{*}$ & Increased \\
\hline Beijing & 4882 & 1.99 & 4.07 & 0.61 & $-24.17(-26.46 \text { to }-21.81)^{*}$ & Decreased \\
\hline Fujian & 10,815 & 1.96 & 0.69 & 2.81 & $8.04(-5.53$ to 23.55$)$ & Stable \\
\hline Hebei & 20,985 & 1.93 & 1.12 & 2.48 & $13.05(10.02 \text { to } 16.16)^{*}$ & Increased \\
\hline Ningxia & 1799 & 1.89 & 1.52 & 2.14 & $3.59(-0.98$ to 8.38$)$ & Stable \\
\hline Henan & 20,589 & 1.45 & 0.43 & 2.13 & $19.31(12.96 \text { to } 26.02)^{*}$ & Increased \\
\hline Gansu & 5087 & 1.31 & 0.35 & 1.94 & $22.75(16.77 \text { to } 29.04)^{*}$ & Increased \\
\hline Qinghai & 894 & 1.04 & 0.64 & 1.3 & $11.42(7.48 \text { to } 15.51)^{*}$ & Increased \\
\hline Tibet & 434 & 1.02 & 1.04 & 1.01 & $1.42(-19.07$ to 27.10$)$ & Stable \\
\hline Shanghai & 2396 & 0.86 & 1.12 & 0.69 & $-22.84(-29.81 \text { to }-15.17)^{*}$ & Decreased \\
\hline Jiangsu & 9398 & 0.8 & 0.48 & 1.02 & $7.97(-6.48$ to 24.65$)$ & Stable \\
\hline Shanxi & 4066 & 0.76 & 0.48 & 0.95 & $3.75(-4.90$ to 13.18$)$ & Stable \\
\hline Tianjin & 1190 & 0.71 & 1.32 & 0.30 & $-23.49(-26.5 \text { to }-20.35)^{*}$ & Decreased \\
\hline Shandong & 8695 & 0.6 & 0.2 & 0.87 & $18.59(9.63 \text { to } 28.29)^{*}$ & Increased \\
\hline Inner Mongolia & 1976 & 0.54 & 0.42 & 0.62 & $12.39(2.3 \text { to } 23.47)^{*}$ & Increased \\
\hline Xinjiang & 1111 & 0.36 & 0.64 & 0.17 & $-15.15(-18.08 \text { to }-12.12)^{*}$ & Decreased \\
\hline Jilin & 1300 & 0.32 & 0.16 & 0.42 & $-3.27(-20.67$ to 17.94$)$ & Stable \\
\hline Liaoning & 1264 & 0.19 & 0.14 & 0.23 & $4.92(-1.42$ to 11.67$)$ & Stable \\
\hline Heilongjiang & 604 & 0.11 & 0.07 & 0.13 & $0.15(-8.10$ to 9.15$)$ & Stable \\
\hline Mainland China & 720,640 & 3.58 & 1.82 & 4.75 & $8.04(-5.53$ to 23.55$)$ & Stable \\
\hline
\end{tabular}

${ }^{*} P<0.05$

low- and middle-income provinces from 2004 to 2018. In addition, there was clear seasonality from August to October and a specific high-risk age group in children. Several sociodemographic factors were identified to be significantly associated with the AHC incidence. These findings will help government develop disease-specific and location-specific interventive measures in the following stage.

Zhang's study reported that higher mean temperature, relative humidity and precipitation were associated with an increased risk of AHC [45]. Meteorological conditions in South China were also characterized by risk factors of high temperature, high relative humidity, and abundant rainfall, especially during summer, which were suitable for the growth and reproduction of intestinal viruses [46]. Therefore, in those high burden key provinces, strengthening epidemic surveillance by monitoring before the epidemic season, timely assessment and early warning of the epidemic development trend are critical for the prevention and control of $\mathrm{AHC}$. This finding revealed that the AHC incidence increased from 2004-2010 but stabilized after 2010. Since 2004, China has established a notifiable infectious disease surveillance/reporting system with continuous standardization and maturity [22]. It has greatly promoted the prevention and control of 40 kinds of infectious diseases in China, including AHC. During 


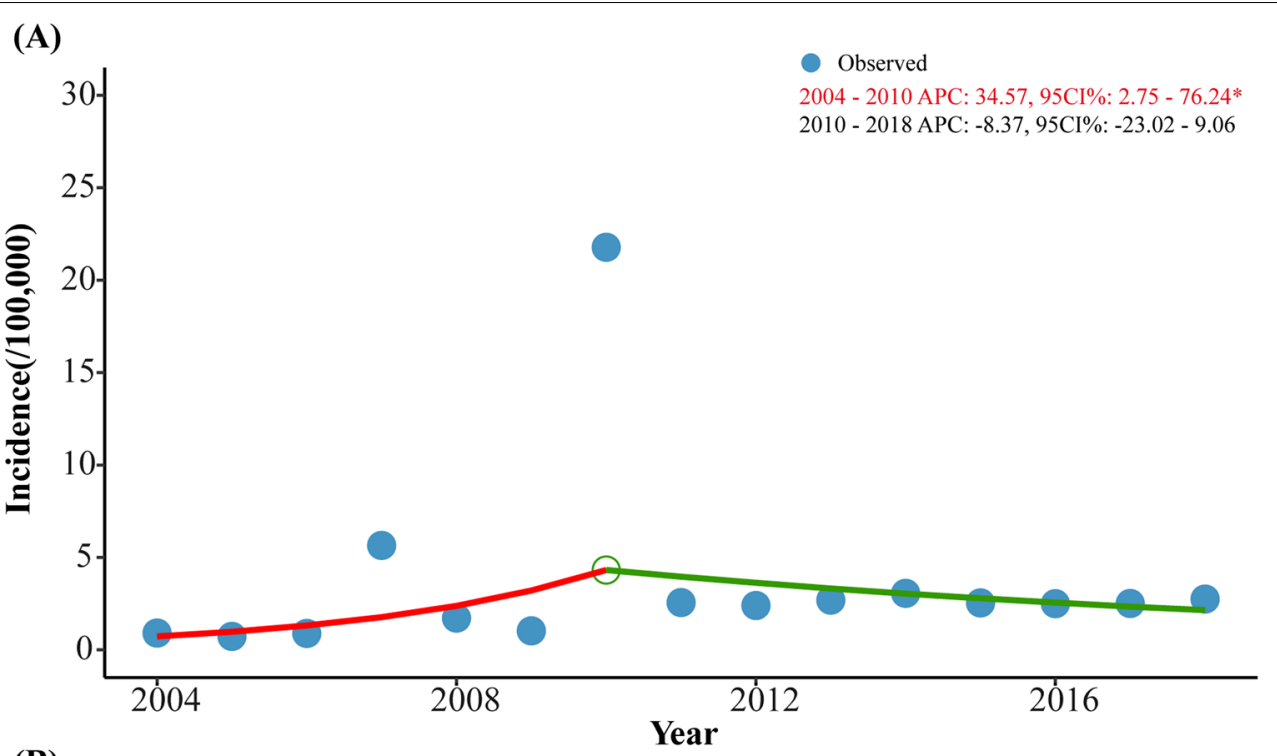

(B) Year

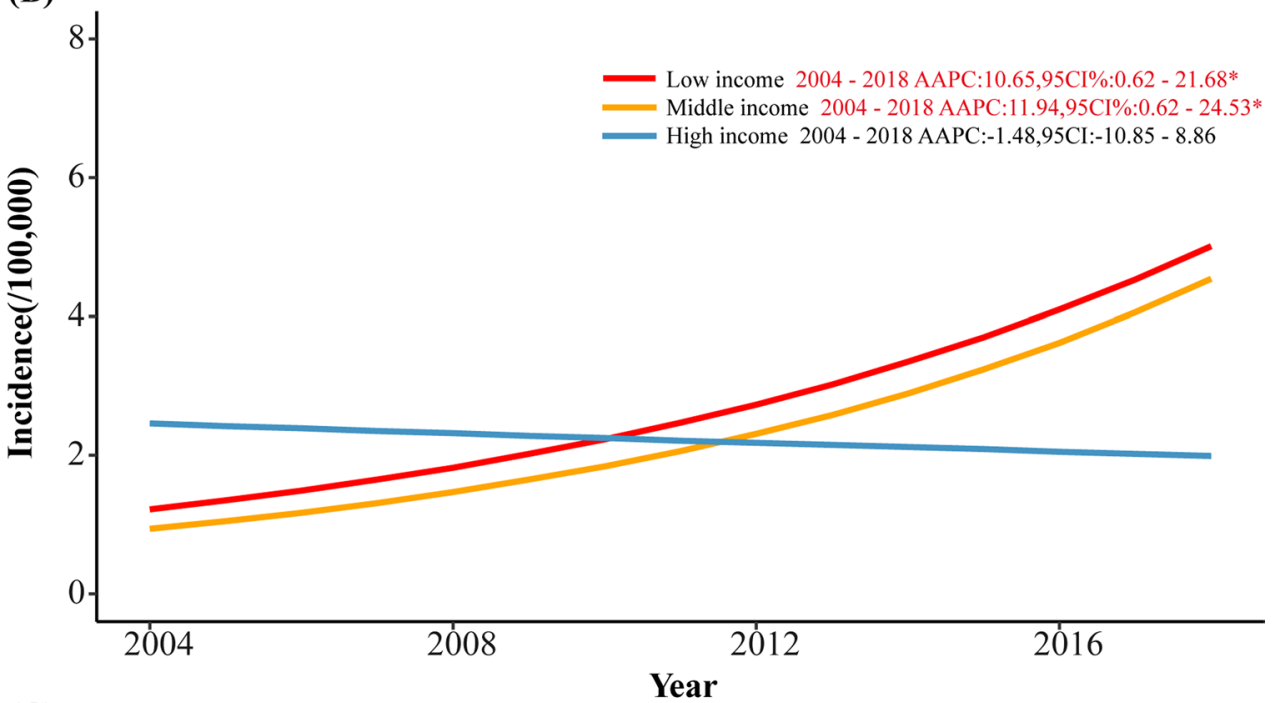

(C)

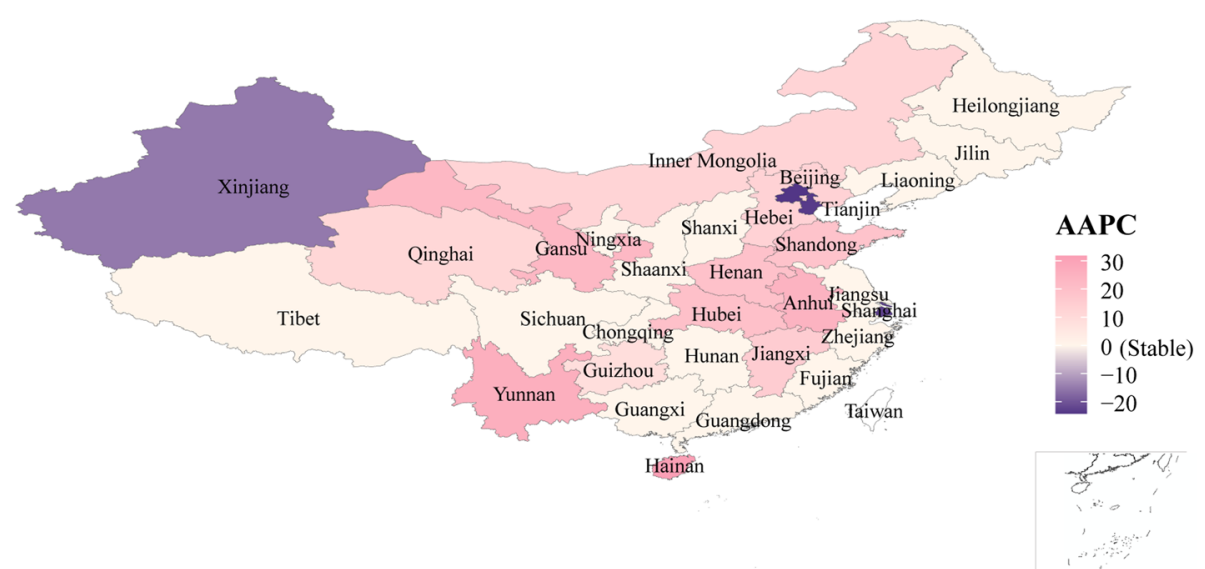

Fig. 2 Trends in incidence of acute hemorrhagic conjunctivitis in mainland China, 2004-2018 (red font*: statistically significant trends; APC: annual percentage change; AAPC: average annual percentage change) 


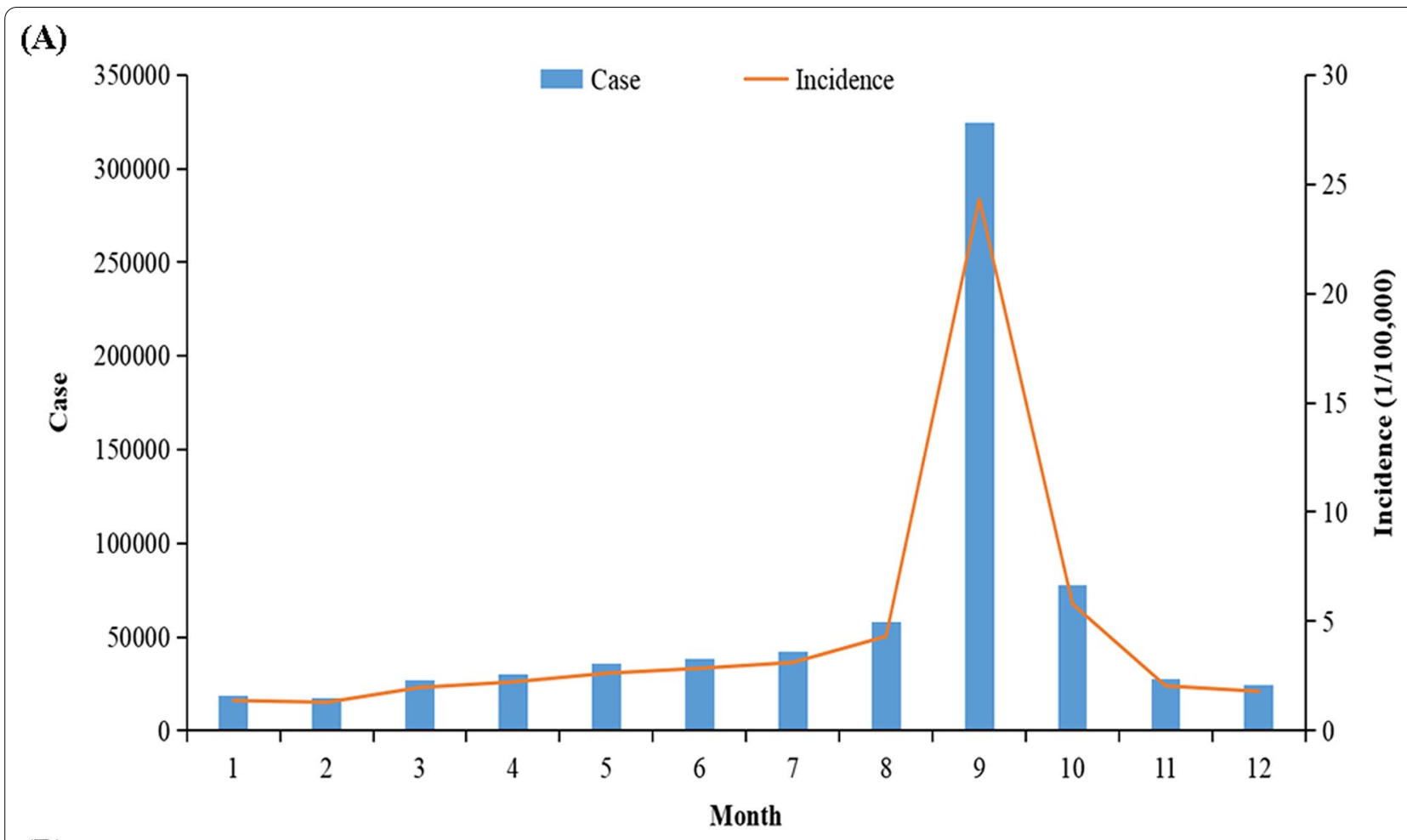

(B)

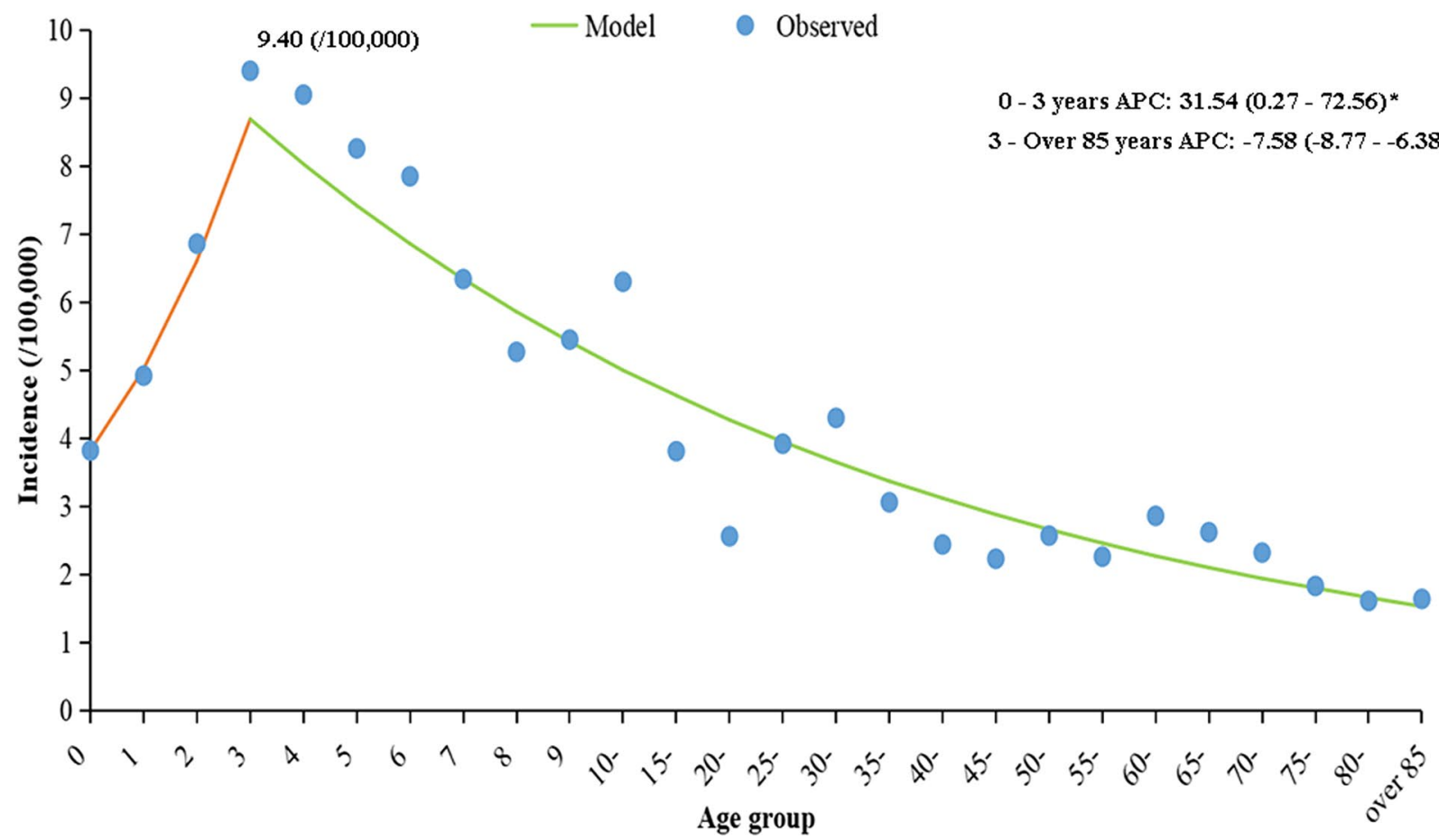

Fig. 3 The seasonality and age group distribution of acute hemorrhagic conjunctivitis in mainland China, 2004-2018

the study period, there were two peaks of AHC incidence, in 2007 and 2010 [17]. Since 1971, Cox24v has been the major pathogen of AHC in China [47]. The outbreaks caused by CA24v are usually more widespread than those caused by EV70 [9]. From 1986 to 1988, CA24v caused epidemics in Beijing, Shanghai, and Guangzhou[48]. In 
Table 2 The log-linear model for the association between sociodemographic factors and incidence of acute hemorrhagic conjunctivitis

\begin{tabular}{lccc}
\hline Variables & Coefficient & Standard error & \multicolumn{1}{c}{ value } \\
\hline Year & 0.1654 & 0.0248 & $<0.001^{* *}$ \\
Birth rate & 18.513 & 4.5161 & $0.001^{* *}$ \\
Population ages 0-14 (\% of total population) & 0.0760 & 0.0376 & $0.044^{*}$ \\
Urban population (\% of total population) & 0.0755 & 0.0119 & $<0.001^{* *}$ \\
Population density & 0.0001 & 0.0002 & 0.704 \\
Log passenger traffic & 0.1244 & 0.0267 & $<0.001^{* *}$ \\
Gross domestic product per capital & -0.0004 & 0.0000 & $<0.001^{* *}$ \\
Health workers (per 1000 people) & -0.0219 & 0.0594 & 0.106 \\
\hline$*$ P<0.001; $P<0.05$ & & &
\end{tabular}

${ }^{*} P<0.001 ; * P<0.05$

2007-2008, it was also responsible for outbreaks of AHC in Zhejiang, Yunnan and Guangdong [33, 49]. Two years later, the intensity of the outbreak in China caused by CA24v was significantly higher than that in 2007 and presented spatial-temporal clusters. G4-c3, G4-c5a and c5b of CA24v were the predominant strains in China from 2004 to 2014. They cocirculated and coevolved in southern and eastern China [16]. The genetic diversity of virus type alternation and antigen variation, lack of cross-protection between different genotypes, and poor immune persistence after infection may have resulted in random outbreaks of AHC [12]. In addition, the incidence of AHC was significantly increased in low- and middleincome provinces during the study period. Lower-income provinces are usually characterized by low immune systems, poor living environments, and poor knowledge of epidemic prevention measures, which might cause AHC to spread easily [50]. Regarding the AAPC, the provinces with higher increasing rates are also noticeable. Continuous surveillance to monitor genotypes and the emergence of new strains for the development of prevention and control strategies is necessary.

Children are usually the most frequently infected group [51]. In addition to school children, our results indicated that young children age 0-3 years are still worth studying. The absence of protective antibodies against enterovirus, low immunity and lack of basic hygiene knowledge in young children, along with poor knowledge of AHC among their parents (kindergarten teachers), leading to the inability to rapidly identify the main clinical symptoms, may result in higher risk $[18,52]$. Therefore, health education should address hand-eye hygiene and improve the ability of parents (kindergarten teachers) to recognize the symptoms of conjunctivitis at an early stage.

Our results also provide insights into the relationship between sociodemographic factors and AHC, whose results emphasize the necessity of incorporating these parameters into the current surveillance system for early warning of AHC. Regions with high birth rates and populations aged $0-14$ (\% of the total population) contribute to high AHC incidence, echoing the results of high-risk age groups in the above discussion. China is rapidly becoming more urbanized, with low- and middle-income provinces experiencing the most striking social structure changes, which are also related to high AHC incidence, similar to other infectious diseases [31, 53, 54]. In addition to urbanization, passenger traffic greatly increases the risks of infectious disease spread, also including AHC [53]. Finally, yet importantly, regions with low per capita gross domestic products are at high risk of AHC, which may be characterized by poor sanitation permitting these causative agents to spread quickly $[18,50]$, echoing the results of the trends of AAPC subgroups by income. Those findings emphasize the necessity of incorporating these parameters into the current surveillance system for early warning of AHC. Characteristics related to the high incidence of AHC can also be applied to the prevention and control of AHC in other countries. Public health authorities should strengthen the surveillance of AHC in regions with these characteristics. Simultaneously, before the high-risk season, it is important to publicize and educate the public on the prevention and control of AHC, especially in high-risk groups. These efforts can help them understand the transmission and prevention methods of AHC, lend attention to personal eye hygiene, and form good hygiene habits and self-protection awareness. In addition, the infected should seek medical treatment as soon as possible and avoid going to public places, such school, nursery, factory, or natatorium [32].

\section{Limitation}

This study has several limitations. First, in surveillance data, underreporting is a common problem. However, the dataset on the basis of the notifiable infectious disease reporting system used in this study was the most complete dataset currently available. Second, 
the sociodemographic factors identified in our study were only correlated with $\mathrm{AHC}$, and a causal relationship was not proven. Further investigation is needed to determine the causality to provide a more precise basis when formulating targeted prevention policies. Last, this research only involved study site in China, more relatedresearches in different countries are also encouraged, then to provide more scientific evidence for prevent and control AHC, as well as other infectious diseases in future.

\section{Conclusion}

$\mathrm{AHC}$ has a clear regional, population, and seasonal distribution in China during 2004-2018. The burden of AHC was high in South China, and children between the ages of $0-3$ were at high risk, while the seasonal peak of AHC prevalence was from August to October. After 2010, the AHC incidence remained stable; however, in low- and middle-income provinces, the AHC incidence continuously increased. Regions with a high birth rate, population ages $0-14$ (\% of the total population), urban population (\% of the total population), passenger traffic and low per capita gross domestic product are at high risk of AHC. In the future, public health policy and resource priority for $\mathrm{AHC}$ in regions with these characteristics are necessary.

\section{Abbreviations}

AHC: Acute hemorrhagic conjunctivitis; APC: Annual percentage change; AAPC: Average annual percentage change; RR: Relative risk; LLR: Log likelihood ratio.

\section{Supplementary Information}

The online version contains supplementary material available at https://doi. org/10.1186/s12985-022-01758-6.

Additional file 1: Table S1. The brief outline of study. Additional file 1: Table S2. Global autocorrelation analysis of acute hemorrhagic conjunctivitis in mainland China, 2004-2018.Additional file 1: Table S3. Bivariate global Moran's I between sociodemographic factors and incidence of acute hemorrhagic conjunctivitis in mainland China, 2004-2018. Additional file 1: Figure S1. Spatial autocorrelation analysis of acute hemorrhagic conjunctivitis in mainland China, 2004-2018.

\section{Acknowledgements}

We thank the Chinese Center for Disease Control and Prevention for opening and sharing the Public Health Science Data Center.

\section{Authors' contributions \\ $\mathrm{RL}$ and FZ designed the study. RL, YC, and $\mathrm{HL}$ collected data. $\mathrm{RL}$, and $\mathrm{YC}$ analyzed data. RL and YC checked the data and results. RL interpreted data and wrote the report. RL and YC revised the report from preliminary draft to submission. XH modified the language. RL and FZ supervised the study. All authors have read and approved the manuscript.}

\section{Funding}

The author(s) received no financial support for the research, authorship, and/ or publication of this article.

\section{Availability of data and materials}

The datasets used and/or analyzed during the current study are available from the corresponding author on reasonable request.

\section{Declarations}

Ethics approval and consent to participate

Not applicable.

\section{Consent for publication}

Not applicable.

\section{Competing interests}

The authors have no conflicts of interest relevant to this article to disclose.

\section{Author details}

${ }^{1}$ Institute for Disaster Management and Reconstruction, Sichuan University, Chengdu 610000, China. ${ }^{2}$ Institute of Chronic and Non-Communicable Disease Control and Prevention, Hubei Provincial Center for Disease Control and Prevention, No. 35 Zhuodaoquan North Road, Hongshan District, Wuhan 430079, China. ${ }^{3}$ Subject Teaching (English), College of Foreign Languages, Fujian Normal University, Fuzhou 350000, China.

Received: 24 October 2021 Accepted: 8 February 2022

Published online: 01 March 2022

\section{References}

1. Burr SE, Sillah A, Joof H, Bailey RL, Holland MJ. An outbreak of acute haemorrhagic conjunctivitis associated with coxsackievirus A24 variant in The Gambia, West Africa. BMC Res Notes. 2017;10:692.

2. National Health Commission of the People's Republic of China. Diagnostic Criteria for Acute Hemorrhagic Conjunctivitis [Internet]. 2008 [cited 2021 Dec 22]. http://www.nhc.gov.cn/wjw/s9491/200802/39044.shtml.

3. Ayoub EA, Shafik CF, Gaynor AM, Mohareb EW, Amin MA, Yassin AS, et al. A molecular investigative approach to an outbreak of acute hemorrhagic conjunctivitis in Egypt, October 2010. Virol J. 2013;10:96.

4. Shukla D, Kumar A, Srivastava S, Dhole TN. Molecular identification and phylogenetic study of coxsackievirus A24 variant isolated from an outbreak of acute hemorrhagic conjunctivitis in India in 2010. Arch Virol. 2013;158:679-84.

5. Yan D, Zhu S, Zhang Y, Zhang J, Zhou Y, Xu W. Outbreak of acute hemorrhagic conjunctivitis in Yunnan, People's Republic of China, 2007. Virol J. 2010;7:138

6. Zhang S, Hu Q, Deng Z, Hu S, Liu F, Yu S, et al. Transmissibility of acute haemorrhagic conjunctivitis in small-scale outbreaks in Hunan Province, China. Sci Rep. 2020;10:119.

7. Lim KH, Yin-Murphy M. An epidemic of conjunctivitis in Singapore in 1970. Singapore Med J. 1971;12:247-9.

8. Baggen J, Hurdiss DL, Zocher G, Mistry N, Roberts RW, Slager JJ, et al. Role of enhanced receptor engagement in the evolution of a pandemic acute hemorrhagic conjunctivitis virus. Proc Natl Acad Sci USA. 2018;115:397-402.

9. Oh M, Park S, Choi Y, Kim H, Lee K, Park W, et al. Acute hemorrhagic conjunctivitis caused by coxsackievirus A24 variant, South Korea, 2002. Emerg Infect Dis. 2003;9:1010-2.

10. Chansaenroj J, Vongpunsawad S, Puenpa J, Theamboonlers A, Vuthitanachot V, Chattakul P, et al. Epidemic outbreak of acute haemorrhagic conjunctivitis caused by coxsackievirus A24 in Thailand, 2014. Epidemiol Infect. 2015;143:3087-93.

11. Enfissi A, Joffret M-L, Delaune D, Delpeyroux F, Rousset D, Bessaud M. Coxsackievirus A24 variant associated with acute haemorrhagic conjunctivitis cases, French Guiana, 2017. Intervirology. 2017;60:271-5.

12. Fonseca MC, Pupo-Meriño M, García-González LA, Resik S, Hung LH, Muné M, et al. Molecular evolution of coxsackievirus A24v in Cuba over 23-years, 1986-2009. Sci Rep. 2020;10:13761.

13. Sousa IP, Burlandy FM, Ferreira JL, Alves JCS, Sousa-Júnior EC, Tavares $\mathrm{FN}$, et al. Re-emergence of a coxsackievirus A24 variant causing acute 
hemorrhagic conjunctivitis in Brazil from 2017 to 2018. Arch Virol. 2019;164:1181-5.

14. Filleul L, Pagès F, Wan G-NC, Brottet E, Vilain P. Costs of conjunctivitis outbreak, Réunion Island, France. Emerg Infect Dis. 2018;24:168-70.

15. Channa R, Zafar SN, Canner JK, Haring RS, Schneider EB, Friedman DS. Epidemiology of eye-related emergency department visits. JAMA Ophthalmol. 2016;134:312-9.

16. Zhang L, Zhao N, Huang X, Jin X, Geng X, Chan T-C, et al. Molecular epidemiology of acute hemorrhagic conjunctivitis caused by coxsackie A type 24 variant in China, 2004-2014. Sci Rep. 2017;7:45202.

17. Wang X, Zhao J, Zhang S, Si B, Wang Y. Acute hemorrhagic conjunctivitis outbreak in China. Dis Surveill. 2014;29:92-7 ((in Chinese)).

18. De W, Huanying Z, Hui L, Corina M, Xue G, Leng L, et al. Phylogenetic and molecular characterization of coxsackievirus A24 variant isolates from a 2010 acute hemorrhagic conjunctivitis outbreak in Guangdong. China Virol J. 2012;9:41.

19. China Center for Disease Control and Prevention. Overview of the national notifiable infectious disease in 2020, China. [cited 2021 Aug 29]. http://www.nhc.gov.cn/jkj/s3578/202103/f1a448b7df7d4760976fea6d5 5834966.shtml.

20. Jing D, Zhao H, Ou R, Zhu H, Hu L, Giri M, et al. Epidemiological characteristics and spatiotemporal analysis of acute hemorrhagic conjunctivitis from 2004 to 2018 in Chongqing, China. Sci Rep. 2020;10:9286.

21. Chen SL, Liu RC, Chen FM, Zhang XX, Zhao J, Chen TM. Dynamic modelling of strategies for the control of acute haemorrhagic conjunctivitis outbreaks in schools in Changsha, China (2004-2015). Epidemiol Infect. 2017;145:368-78.

22. Yang S, Wu J, Ding C, Cui Y, Zhou Y, Li Y, et al. Epidemiological features of and changes in incidence of infectious diseases in China in the first decade after the SARS outbreak: an observational trend study. Lancet Infect Dis. 2017;17:716-25.

23. The Public Health Science Data Center. China center for disease control and prevention. [cited 2021 Aug 29]. https://www.phsciencedata.cn/ Share/index.jsp.

24. Chinese Center For Disease and Control. Acute hemorrhagic conjunctivitis surveillance. 2005 [cited 2021 Dec 22]. https://www.chinacdc.cn/jkzt/ crb/bl/jxcxxjmy/jc/200509/t20050929_24798.html.

25. Dong $Y$, Wang $L$, Burgner DP, Miller JE, Song $Y$, Ren $X$, et al. Infectious diseases in children and adolescents in China: analysis of national surveillance data from 2008 to 2017. BMJ. 2020:369:1043.

26. Harada K, Fujimoto T, Asato Y, Uchio E. Virological and epidemiological analysis of coxsackievirus A24 variant epidemic of acute hemorrhagic conjunctivitis in Okinawa, Japan, in 2011. Clin Ophthalmol. 2015;9:1085-92.

27. Marguerite N, Brottet E, Pagès F, Jaffar-Bandjee MC, Schuffenecker I, Josset $L$, et al. A major outbreak of conjunctivitis caused by coxsackievirus A24, Réunion, January to April 2015. Euro Surveill. 2016;21:52.

28. Chen T, Liu R. Study on the efficacy of quarantine during outbreaks of acute hemorrhagic conjunctivitis outbreaks at schools through the susceptive-infective-quarantine-removal model. Zhonghua Liu Xing Bing Xue Za Zhi. 2013;34:75-9 ((in Chinese)).

29. Edmonds A, Haley DF, Tong W, Kempf M-C, Rahangdale L, Adimora AA, et al. Associations between population density and clinical and sociodemographic factors in women living with HIV in the Southern United States. AIDS Care. 2021;33:229-38.

30. Yin $H$, Sun T, Yao L, Jiao Y, Ma L, Lin L, et al. Association between population density and infection rate suggests the importance of social distancing and travel restriction in reducing the COVID-19 pandemic. Environ Sci Pollut Res Int. 2021;28:40424-30.

31. Coker RJ, Hunter BM, Rudge JW, Liverani M, Hanvoravongchai P. Emerging infectious diseases in southeast Asia: regional challenges to control. Lancet. 2011;377:599-609.

32. Liu H, Li C, Shao Y, Zhang X, Zhai Z, Wang X, et al. Forecast of the trend in incidence of acute hemorrhagic conjunctivitis in China from 2011-2019 using the Seasonal Autoregressive Integrated Moving Average (SARIMA) and Exponential Smoothing (ETS) models. J Infect Public Health. 2020;13:287-94

33. Wu B, Qi X, Xu K, Ji H, Zhu Y, Tang F, et al. Genetic characteristics of the coxsackievirus A24 variant causing outbreaks of acute hemorrhagic conjunctivitis in Jiangsu, China, 2010. PLoS ON. 2014;9:e86883.
34. Faria NR, Rambaut A, Suchard MA, Baele G, Bedford T, Ward MJ, et al. HIV epidemiology. The early spread and epidemic ignition of HIV-1 in human populations. Science. 2014;346:56-61.

35. Hao Y, Zhang N, Wu J, Su B, Gong L, Ma W, et al. Identifying infectious diarrhea hot spots and associated socioeconomic factors in Anhui Province. China Am J Trop Med Hyg. 2019;101:549-54.

36. Chongsuvivatwong V, Phua KH, Yap MT, Pocock NS, Hashim JH, Chhem $\mathrm{R}$, et al. Health and health-care systems in southeast Asia: diversity and transitions. Lancet. 2011;377:429-37.

37. Kulldorff M, Huang L, Pickle L, Duczmal L. An elliptic spatial scan statistic. Stat Med. 2006;25:3929-43.

38. SaTScan-Technical Documentation [Internet]. [cited 2021 Aug 29]. https://www.satscan.org/techdoc.html.

39. Thompson ES, Saveyn P, Declercq M, Meert J, Guida V, Eads CD, et al. Characterisation of heterogeneity and spatial autocorrelation in phase separating mixtures using Moran's I. J Colloid Interface Sci. 2018;513:180-7.

40. Cheng Z The spatial correlation and interaction between manufacturing agglomeration and environmental pollution. Ecol Ind. 2016;61:1024-32.

41. Parra-Amaya ME, Puerta-Yepes ME, Lizarralde-Bejarano DP, ArboledaSánchez S. Early detection for dengue using local indicator of spatial association (LISA) analysis. Diseases. 2016;4:E16.

42. Kim HJ, Fay MP, Feuer EJ, Midthune DN. Permutation tests for joinpoint regression with applications to cancer rates. Stat Med. 2000;19:335-51.

43. National Burea of Statistics. China Statistics Yearbook (2019). 2019 [cited 2021 Dec 23]. http://www.stats.gov.cn/tjsj/ndsj/.

44. Scott M, Flaherty D, Currall J. Statistics: general linear models (a flexible approach). J Small Anim Pract. 2014;55:527-30.

45. Zhang L, Jiang H, Wang K, Yuan Y, Fu Q, Jin X, et al. Long-term effects of weather condition and air pollution on acute hemorrhagic conjunctivitis in China: a nationalwide surveillance study in China. Environ Res. 2021:201:111616.

46. Liu X, Qiu S, Liu Z, Chen D, Liu H, Ding G. Effects of floods on the incidence of acute hemorrhagic conjunctivitis in Mengshan, China, from 2005 to 2012. Am J Trop Med Hyg. 2020;102:1263-8.

47. Zhao Y, Liu J, Zhang H, Guo C, Xia L, Yang F, et al. Complete genome analysis of coxsackievirus A24 isolated in Yunnan, China, in 2013. Arch Virol. 2016;161:1705-9.

48. Yan J, Chen Y, Li Z, Gong L, Lu Y, Zhang Y. Study on the etiology of acute hemorrhagic conjunctivitis outbreak in some areas of Zhejiang Province in 2010. Chin J Virol. 2011;27:421-6 ((in Chinese)).

49. Wu D, Ke C-W, Mo Y-L, Sun L-M, Li H, Chen Q-X, et al. Multiple outbreaks of acute hemorrhagic conjunctivitis due to a variant of coxsackievirus A24: Guangdong, China, 2007. J Med Virol. 2008;80:1762-8.

50. Mao Y, Zhang N, Zhu B, Liu J, He R. A descriptive analysis of the Spatiotemporal distribution of intestinal infectious diseases in China. BMC Infect Dis. 2019;19:766.

51. Chawla R, Kellner JD, Astle WF. Acute infectious conjunctivitis in childhood. Paediatr Child Health. 2001;6:329-35.

52. Wang $X$, Zhao J, Zhang S, Si B, Wang Y. Acute hemorrhagic conjunctivitis outbreak in China. Dis Surveill. 2014;29:92-7 ((in Chinese)).

53. Ra W, Aj M. Social and environmental risk factors in the emergence of infectious diseases. Nat Med. 2004;10.

54. National Burea of Statistics. China statistics yearbook (2005-2019). 2019. http://www.stats.gov.cn/tjjj/ndsj/.

\section{Publisher's Note}

Springer Nature remains neutral with regard to jurisdictional claims in published maps and institutional affiliations. 